\title{
Simulation and application of transformer with multi-magnetic circuits in the ship-breaker's verifying equipment \\ ${ }^{1, a}$ Wang Zongliang, ${ }^{2, b}$ Sun Junzhong, ${ }^{3, c}$ Yang Zhanlu \\ ${ }^{1,2}$ Naval Submarine Academy, Qingdao 266042, China \\ a,b,c qiantingwzl@163.com
}

Keywords: Breaker; verifying equipment; MATLAB; transformer with multi-magnetic circuits; simulation.

Abstract. According to the working characteristic of the transformer with multi-magnetic circuits, a simulation model of the transformer with multi-magnetic circuits is established, by using of simulation model and SD-3 type monitor in the ship-breaker's verifying equipment, close making angle can be selected quickly and exactly in instantaneous test.

\section{Introduction}

Ship-breaker' verifying equipment (hereinafter referred to as verifying equipment) as the important equipment to test operational reliability, it requires to make stable smooth and significant adjustment so as to guarantee the precision of verifying. The traditional method of large current output has several models such as combination of voltage regulator and large-current transformer, voltage regulator of thyristor and voltage regulator of magnetic. As for breaker verifying equipment of large capacity, the direct application of large-capacity breaker verifying equipment exists problems of huge volume, higher cost, big voltage drop and low overload capacity etc, while the other 2 kinds of method have problems such as higher harmonic, disturbance power grid, this is not allowed by verifying equipment. In view of the above reasons, in order to obtain enough big current output capacity by economic and reasonable methods as well as adapt to strong current test requirement of ship-breaker with large capacity, the verifying equipment adopts system of transformer with multi-magnetic circuits.

\section{Transformer with multi-magnetic circuits}

Transformer with multi-magnetic circuits is composed of 4 different magnetic of common reactance:, including 2 small magnetic circuit of $1 / 6$ output and 2 big magnetic circuit of 1/3 output, it will optionally control input of magnetic circuit by input terminal, it can use voltage of output terminal to increase by grade of 1:2:3:4:5:6. However, access one voltage regulation with the same capacity in the magnetic circuit of producing 1/6 output, so that it can make stepless continual voltage regulation within each grade range, so it further gets effect of grading and adjustable in the whole process.

According to the operational characteristics of transformer with multi-magnetic circuits and maximum current provided by ship-breaker verifying equipment, this paper designs one set of transformer system with total capacity of 60KVA, capacity of each magnetic circuit is respectively 20KVA, 10KVA, 10KVA, 20KVA, the rated work voltage is AC $380 \mathrm{~V}$, of which, the transformation ratio of 10KVA magnetic circuit is 142:1, the transformation ratio of 20KVA magnetic circuit is 142:2, connection of both arms adopts series connection.

\section{Overall simulation of transformer with multi-magnetic circuits}

Simulation model of each magnetic:This paper chooses MATLAB/SIMULINK as the simulation platform towards system. Because verifying equipment is mainly big current test of short time 
under failure condition of breaker, which makes transformer of multi-magnetic circuits work under overload state and the overload current born by transformer of multi-magnetic circuits with different characteristics is also different. Therefore, in order to precisely and roundly reflect the temporary steady process of transformer with multi-magnetic circuits in the test process, simulation of each magnetic chooses single-phase transformer model.

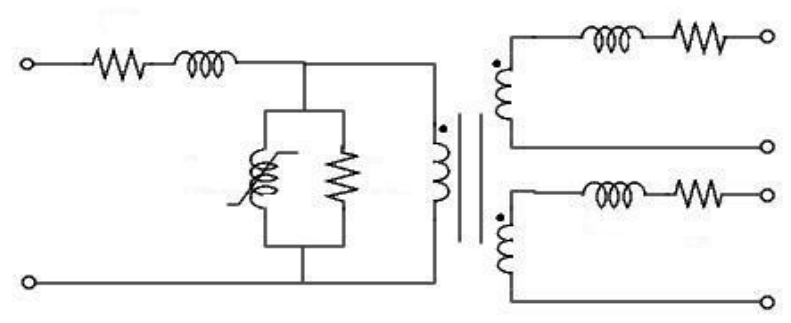

Diagram.1: Single-phase saturated transformer

Circuit diagram of single-phase saturated transformer is indicated by diagram 1 , in the models, R1 and L1 are resistance and leakage inductance of primary winding, R2, L2 and R3, L3 are respectively resistance and leakage inductance of secondary winding, magnetic resistance $\mathrm{Rm}$ is equal to the core loss of transformer, Lsat is used to reflect saturated characteristics of core. Model parameter and settings are indicated in table 1.

Table 1: Model parameter and settings of single=phase saturated transformer

\begin{tabular}{|c|c|c|c|c|}
\hline $\begin{array}{l}\text { Parameter of } \\
\text { transformer }\end{array}$ & single-phase saturated & Unit & Setting & \\
\hline Rated capacity Pn & Frequency fn & VA, Hz & {$[10 \mathrm{e} 35]$} & {$[20 \mathrm{e} 3$} \\
\hline & Rated voltage U1 & V (effective value) & 380 & 380 \\
\hline Winding 1 & Resistance R1 & 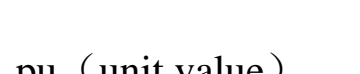 & 0.002 & 0.002 \\
\hline & leakage inductance L & pu (umil value? & 0.09 & 0.09 \\
\hline Winding 2 & Rated voltage U2 & V (unit value) & 2.6 & 4.8 \\
\hline ( secondary side & Resistance R2 & & 0.002 & 0.002 \\
\hline 1) & leakage inductance L2 & pu (unit value) & 0.09 & 0.09 \\
\hline Winding 3 & Rated voltageU3 & V (unit value) & / & / \\
\hline ( secondary side & ResistanceR3 & ) (unit unlus & / & / \\
\hline 2) & leakage inductance L3 & pu cumil varue? & / & / \\
\hline $\begin{array}{l}\text { Saturated } \\
\text { characteristic }\end{array}$ & $\begin{array}{l}\text { magnetization currenti1 } \\
\text { magnetic flow phi1 }\end{array}$ & pu (unit value ) & $\begin{array}{l}{[0,0 ;} \\
0.0024,1.2 ; \\
1.0,1.52]\end{array}$ & $\begin{array}{l}{[0,0 ;} \\
0.0024,1.2 ; \\
1.0,1.52]\end{array}$ \\
\hline $\begin{array}{l}\text { Magnetic } \\
\text { resistance } \\
\text { surplus flux }\end{array}$ & Rm phi0 & pu (unit value ) & {$[500]$} & {$[500]$} \\
\hline
\end{tabular}

Equivalent model of over-current release: In the overall simulation model, over-current release of breaker is equals to the series connection combination of resistance and inductance, its equivalent parameters can be tested and calculated by test. This paper uses transformer with multi-magnetic circuits, power meter, voltage meter and current meter to make test on certain breaker(rated current of over current release is $1500 \mathrm{~A}$ ), the test circuit is indicated by diagram 2 . Firstly, it produces rated current of $1500 \mathrm{~A}$ by adjusting transformer with multi-magnetic circuits, then it tests voltage and consumed active power on both sides of breaker' over-current release under condition of testing this current. It gets the following by test: $\mathrm{P}=450$ watt, $\mathrm{U}=0.6 \mathrm{~V}$, the anti-resistance parameter of breaker' over-current release through calculation is as follows:

$$
Z=0.0004 \Omega, \quad r=0.0002 \Omega, \quad x=3.46 e^{-4} \Omega, \quad L=1.1 e^{-6} \mathrm{H} \text { 。 }
$$




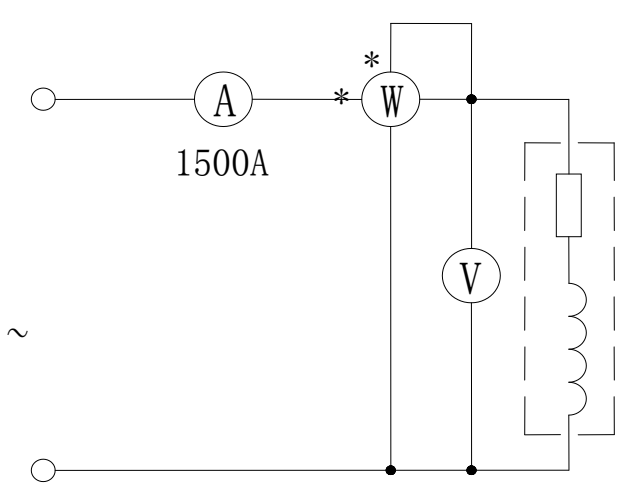

Diagram .2: $\quad$ Test diagram of over-current release

Overall simulation model of transformer with multi-magnetic circuits: After models of each magnetic and over-current resale are confirmed, the main frame of transformer simulation with multi-magnetic circuits is basically formed, the overall model diagram is indicated by diagram 3.

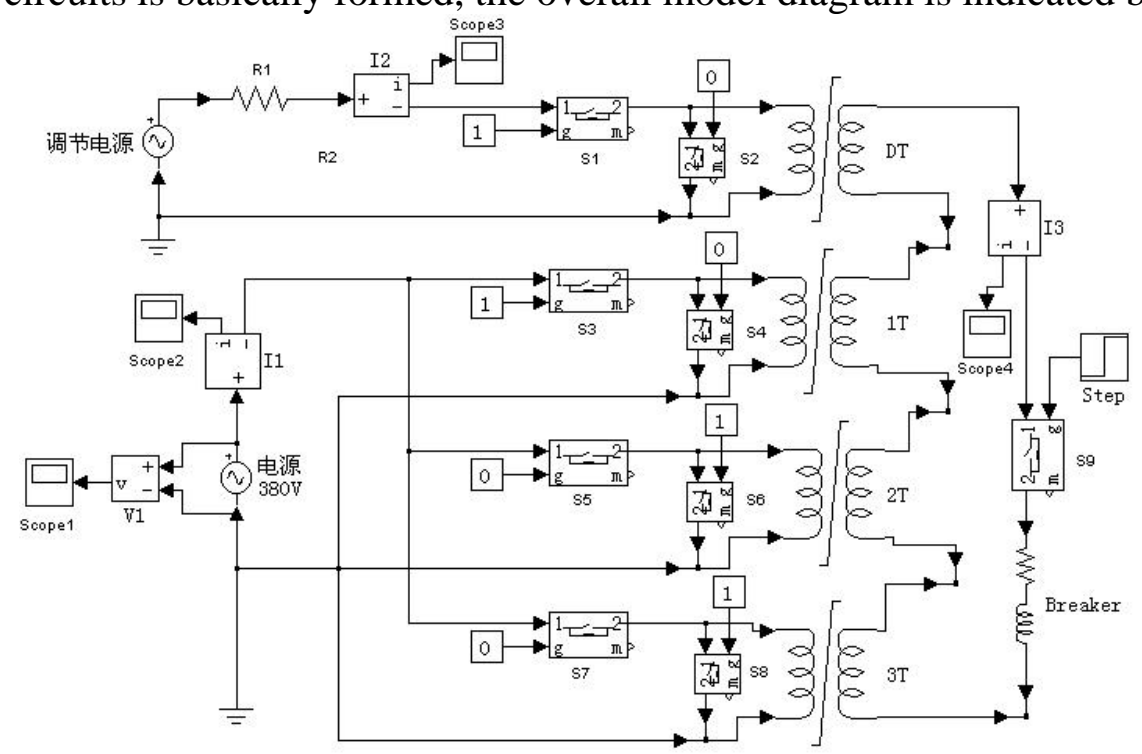

Diagram. 3: Overall simulation model of transformer with multi-magnetic circuits

In order to achieve the same effect with actual test, simulation model adopts the following measures in the establishment process:

( 1 ) There is one independent AC voltage source model of magnetic circuit DT of 1/6 output, it can achieve the same control effect with voltage regulation by changing voltage of voltage source, meanwhile, by changing phase position of voltage source, it can provide current simulation of random angles for angle of optional phase in the short-circuit instantaneous test.

(2) In order to realize switch of transformer with multi-magnetic circuits, simulation model adopts combination of ideal switch for each magnetic circuit and constant module.

(3) In the test loop, in order to correctly control the duration of test current, simulation model adopts combination of step model and ideal switch to change step of step signal then it can correctly control duration of test current.

\section{Application of simulation in phase close}

In the instantaneous test on short-circuit of breaker, because of randomness of close phase-angle, there is plenty of non-periodic components in the test current, and non-periodic component will be different with difference of close angle, which causes asymmetry of current in the primary stage of testing current. Diagram 4 is the current simulation curve of adjusting power $220 \mathrm{~V}$, phase $25^{\circ}$, and power voltage $380 \mathrm{~V}$, phase $25^{\circ}$ on input of transformer DT with multi-magnetic circuits, IT magnetic circuit. 


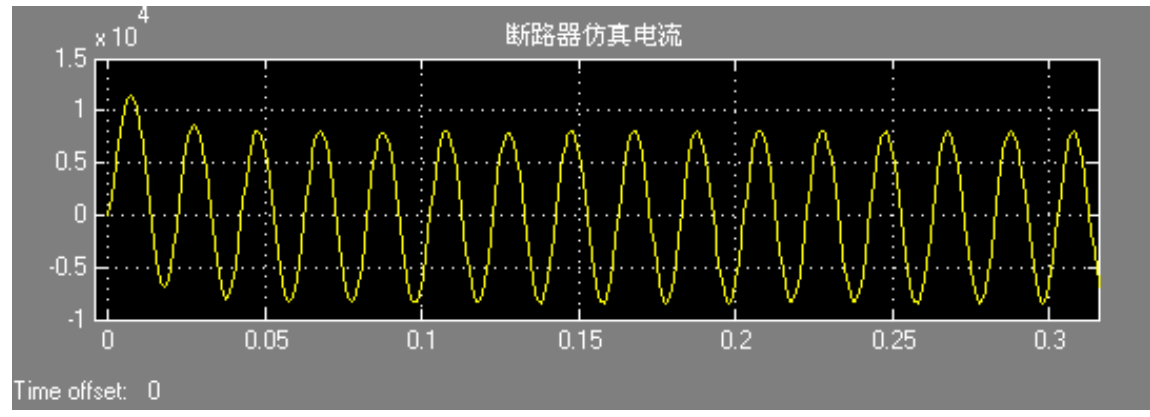

Diagram .4: Current simulation curve of on power phase 25 degree transformer DT with multi-magnetic circuits and IT magnetic circuit into use

Through theoretical analysis, when power loop suddenly closes, the current $i(t)$ comes by over-current release

$i(t)=\frac{E_{m}}{\sqrt{R^{2}+\omega^{2} L^{2}}} \sin (\omega t+\alpha-\varphi)-\frac{E_{m}}{\sqrt{R^{2}+\omega^{2} L^{2}}} \sin (\alpha-\varphi) e^{-\frac{R}{L} t}$

Of which, $\alpha$ is close phase angle of power, $\varphi$ is power factor angle of test loop, $E_{m}$ is voltage amplitude value of test loop

From the above formula we can see that test current includes 2 components, of which the first item is stable periodic component, the second item is non-periodic component with gradual reduction, and non-periodic component is related to close phase-angle of power loop. When close angle of power loop is equal to power factor angle in test loop, non-periodic component is 0 , test current is standard sinusoidal wave. Therefore, in the instantaneous test of breaker short-circuit, in order to reduce or eliminate non-periodic component in test current, the verifying equipment chooses technology of phase close, it guarantees distortion factor of test current is less than $5 \%$ by instantaneous measurement and control apparatus of SD-3 to realize correct close of power loop under the given phase-angle.

However, instantaneous measurement and control apparatus of SD-3 can only set phase-angle time-delay to realize choice of close angle of power loop by manual under condition of already known factor of test loop power. If it is difficult to test power factor, it can only use method of test one by one, and then finds suitable close angle according to power wave of data collection system. Obviously, this method has low efficiency and frequent operation; it will cause certain damage for test equipment.

For this, verifying equipment makes comprehensive application for simulation model of transformer with multi-magnetic circuits and instantaneous measure and control apparatus of SD-3. Firstly, through current simulation on each close angle, it can quickly find close angle which conforms to requirement under simulation condition, then applies it into close angle of instantaneous measure and control apparatus, then demonstrates whether close angle under simulation condition according to current wave of data collection system. This method of first simulation and then application not only has higher efficiency, precise phase choice and it effectively reduces damages on test circuit and equipment. In instantaneous test of certain breaker, this paper uses this method to quickly and precisely confirm that test current basically achieves standard sinusoidal wave when power loop is closed when phase angle is $75^{\circ}$. Diagram 5 is the simulation current wave of transformer with multi-magnetic circuit when power phase angle is closed on $75^{\circ}$, diagram 6 is the test current wave by data collection system when close angle of instantaneous measure and control apparatus of SD-3 is 75 degree. 


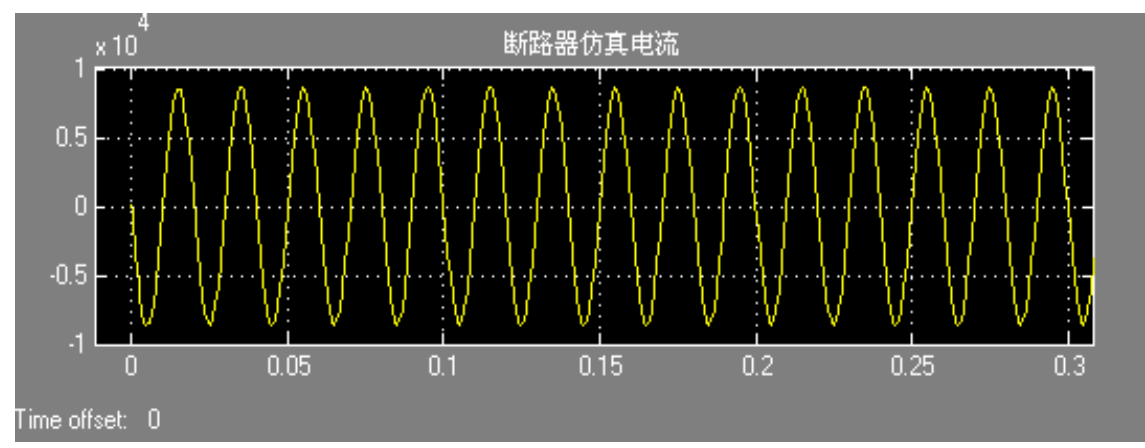

Diagram. 5: Simulation current wave closed on 75 degree

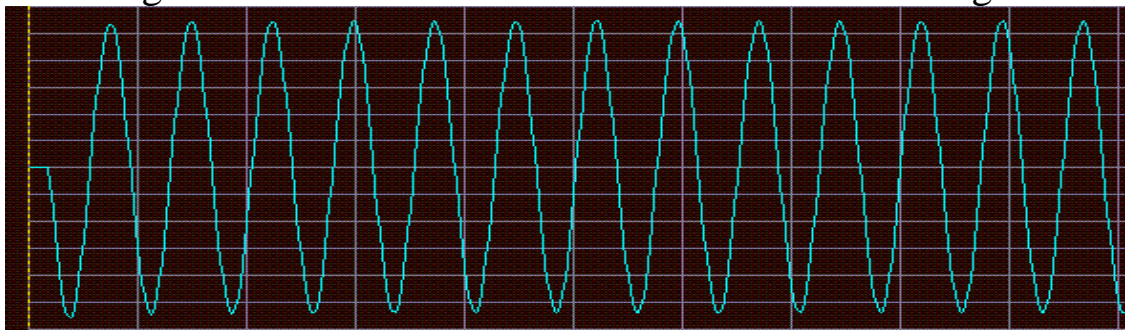

Diagram .6: Test current wave of close on 75 degree

\section{Conclusions}

Through comparison of simulation and actual test, the simulation model established by this paper can correctly reflect temporary steady process of transformer with multi-magnetic circuits in the switch process, and it enhances choice speed and precision of power loop close angle, reduces operation frequency and duration of big current of verifying equipment through comprehensive application of transformer with multi-magnetic circuits and instantaneous measure and control apparatus of SD-3.It reduces power loss of system, and meanwhile lengthens service life of equipment.

\section{References}

[1] Fei Rangruo. Transformer with Multi-magnetic Circuits - One Kinds of New Voltage Regulation System with Big Power [J].Transformer, 2000 (10).

[2] Xi Yongyuan, Shi Jiwei, Du Xiaoping. Verifying Technology on Characteristics of Breaker Action [J]. Low-voltage apparatus, 1996 (6).

[3] Zhang Zheng. Verifying Method and Equipment Analysis on Instantaneous Release Characteristics [J]. Low-voltage apparatus, 2000 (1).

[4] Hong Naigang etc. Power Electronics and MATLAB Simulation of Electrical Towage Control Systems [M].Beijing: Machine Press, 2006.05. 